[0212-7199 (2005) 22: 5; pp 241-243] ANALES DE MEDICINA INTERNA Copyright $(2) 2005$ ARAN EDICIONES, S.L

AN. MED. INTERNA (Madrid) Vol. 22, N. ${ }^{\circ}$ 5, pp. 241-243, 2005

\title{
Prótesis mecánica mitral y cuadros presincopales
}

\author{
E. ARANA RUEDA, F. FLORIÁN SANZ, C. GÓMEZ NAVARRO, J. NEVADO \\ PORTERO, A. AGUILERA SABORIDO, F. LÓPEZ PARDO
}

Servicio de Cardiología. Hospital Universitario Virgen del Rocío. Sevilla

\author{
MITRAL PROSTHETIC VALVE AND PRESYNCOPE
}

\section{RESUMEN}

La trombosis protésica mitral es una entidad poco frecuente, cuya incidencia se relaciona habitualmente con niveles bajos de anticoagulación. La presentación clínica varía desde pacientes asintomáticos, embolismo arterial o compromiso hemodinámico por obstrucción. Presentamos el caso de una disfunción protésica mitral con cuadros presincopales por obstrucción intermitente al flujo.

PALABRAS CLAVE: Válvulas cardiacas mecánicas. Válvula mitral. Trombosis. Síncope.

\begin{abstract}
The incidence of mitral prosthetic valve thrombosis is low and generally dependent on low levels of anticoagulation. The clinical presentation is highly variable and range from asintomatic patient though to arterial embolism or hemodinamic symptoms of valve obstruction.

We report a case of a patient with presyncope and prosthetic mitral valve dysfunction with intermitent flow obstruction.
\end{abstract}

KEY WORDS: Mechanic heart valves. Mitral valve. Thrombosis. Syncope.

Arana Rueda E, Florián Sanz F, Gómez Navarro C, Nevado Portero J, Aguilera Saborido A, López Pardo F. Prótesis mecánica mitral y cuadros presincopales. An Med Interna (Madrid) 2005; 22: 241-243.

\section{INTRODUCCIÓN}

En la valoración clínica del presincope/sincope, es la presencia de cardiopatía estructural el principal determinante pronóstico y la que nos va a orientar a realizar unos u otras pruebas diagnósticas (1). La presentación clínica de la trombosis protésica mitral con cuadros presincopales aislados, es rara (6), pudiendo existir una alta sospecha clínica con métodos diagnósticos sencillos (7). Presentamos el caso de una disfunción protésica mitral manifestada como episodios presincopales frecuentes.

\section{CASO APORTADO}

Mujer de 53 años con antecedentes de enfermedad mitral reumática en fibrilación auricular crónica y con reemplazo valvular por prótesis metálica monodisco del número 29 . Un mes tras el implante presenta un AVC isquémico parietal con recuperación total, coincidiendo con infradosificación de la anticoagulación oral (INR $<2)$.
Posteriormente la paciente presentaba episodios diarios presincopales, acompañados de crisis de palpitaciones y con recuperación a la normalidad en segundos, con frecuencia progresivamente mayor y predominando al adoptar el decúbito. Inicialmente se orientó como crisis comiciales secundarias a lesión isquémica cerebral, recibiendo tratamiento con fenitoina de modo empírico, pendiente de estudio neurológico. Acude a urgencias por presentar constantemente cuadros similares. A la exploración la paciente estaba asintomática, con tensión arterial de 130/80, presentaba tonos arrítmicos a unos 110 latidos por minuto, estando el ruido protésico conservado y sin soplos significativos. Destacaba la ausencia de ruido protésico y pulso periférico de segundos de duración con posterior normalización, coincidiendo con los cuadros de bajo gasto (Fig. 1).

El electrocardiograma y la radiografía de tórax no mostraban datos de interés. Analíticamente el control de anticoagulación mostraba un INR de 1,6 y los niveles de fenitoina estaban en rango terapéutico.

Con la sospecha clínica de disfunción protésica se realizó ecocardiografía transtorácica y posteriormente transesofágica, que mostró una función ventricular conservada, con dilatación leve de ventrículo derecho y biauricular. La prótesis mitral presentaba una apertura

Trabajo aceptado: 20 de diciembre de 2004

Correspondencia: Eduardo Arana Rueda. C/ Virgen de la Antigua 5, duplicado 7-B. 41011 Sevilla. e-mail: eduaru@hotmail.com 


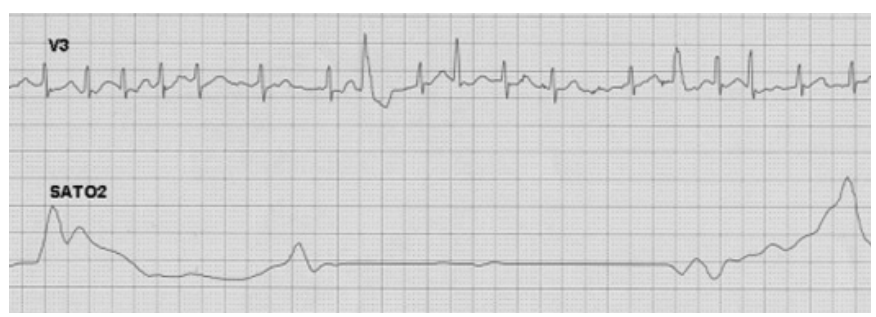

Fig. 1. Trazado de registros electrocardiográfico y pulsioxímetro. Se aprecia falta de correspondencia entre ambos con ausencia de pulso mayores de 4 seg.

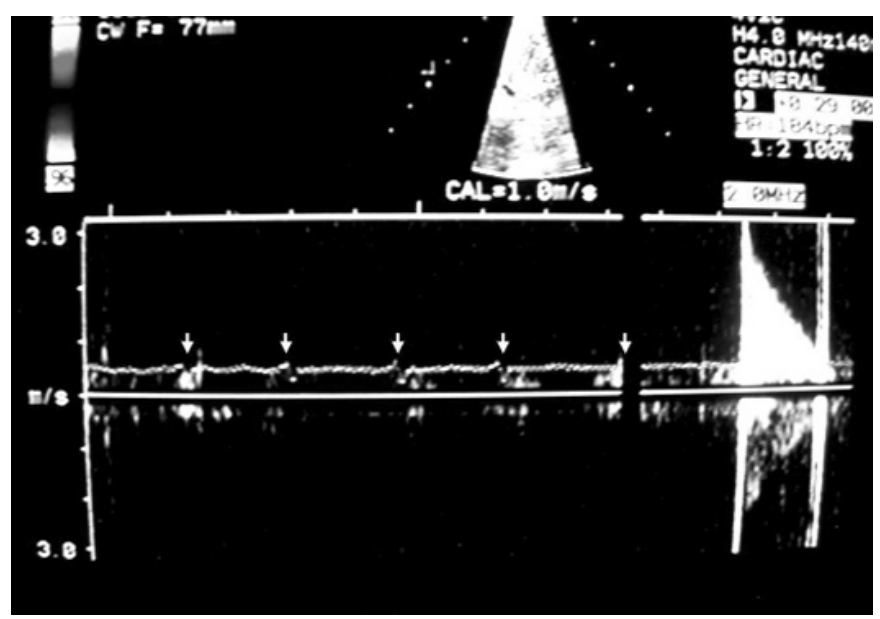

Fig. 2. Registro doppler pulsado mitral Se aprecia mismo fenómeno que en imagen 1, con ausencia de flujo de varios segundos seguida de apertura normal protésica con flujo aumentado (Velocidad máxima de $3 \mathrm{~m} / \mathrm{s}$ ).

conservada, sin regurgitación patológica y con fenómenos de "encasquillamiento" en los que se apreciaba ausencia de flujo (Fig. 2), persistiendo actividad eléctrica y contracción ventricular, seguidos de apertura normal protésica con aumento de flujo a su través (Velocidad máxima de $3 \mathrm{mt} / \mathrm{seg}$ y pendiente aguda) acompañados de los síntomas de bajo gasto periféricos (ausencia de pulso y desaturación en pulsioxímetro). Además se evidenció la presencia de autocontraste severo y trombos en orejuela izquierda, aunque sin extensión a la prótesis. Había también una regurgitación tricuspídea leve sin elevación de presiones derechas.

Con el diagnóstico de trombosis-disfunción protésica, se optó por anticoagulación intravenosa y ante la contraindicación para fibrinolisis y bajo riesgo quirúrgico, a reemplazo valvular por prótesis mecánica bivalva. La intervención cursó sin incidencias y confirmó la presencia de trombos auriculares y posible limitación de la apertura valvular por estructuras miocárdicas, lo que reflejaría una posible interferencia a la apertura por restos de la intervención. La evolución posterior fue favorable.

\section{DISCUSIÓN}

Aunque estudios recientes señalan al presíncope como una entidad con la misma importancia pronóstica que el sín- cope en pacientes con cardiopatía (3), hay dificultades tanto para definir desde su diagnóstico, hasta su significado o tratamiento. La principal diferencia con el síncope radica en la ausencia de perdida de conciencia, siendo los síntomas descritos similares a los de la fase prodrómica de este (mareos, visión borrosa, sensación de perdida inminente de la conciencia...) $(1,2)$. Con una valoración clínica adecuada y técnicas diagnósticas sencillas (analítica, electrocardiograma y ecocardiograma) se pueden orientar tanto el diagnóstico como el pronóstico del síncope en la mayoría de los casos (2). En el diagnóstico diferencial con cuadros convulsivos, que desde el principio se manifiestan con movimientos anómalos, es la perdida de conciencia inicial seguida, tras varios segundos, de convulsiones por hipoperfusión cerebral la que marca la diferencia (crisis de Stokes Adams) (4).

El ecocardiograma, aunque necesario muchas veces para confirmar la presencia de cardiopatía, pocas veces es diagnóstico de la causa del síncope, siendo la disfunción protésica una de ellas $(5,6)$.

Las válvulas protésicas se pueden obstruir por formación de trombos (lo más frecuente), interferencia de tejido fibroso, vegetaciones o estructuras cardiacas, y deterioro o degeneración en el caso de las bioprótesis (6).

Dadas las mejoras técnicas en el desarrollo de prótesis mecánicas y las más adecuadas pautas de anticoagulación, la incidencia de disfunción protésica es rara, siendo del orden del 0,2 a $6 \%$ paciente/año en posición mitral, y fundamentalmente debida a cifras bajas de anticoagulación $(5,6)$. Clínicamente se manifiesta como congestión pulmonar, síntomas de hipoperfusión, como en nuestro caso, o con tromboembolismo arterial.

Los datos exploratorios más llamativos son la disminución del ruido protésico o la aparición de nuevos soplos (57).

En el caso de prótesis monodisco, como el que nos ocupa, los síntomas pueden ser más llamativos, ya que se afecta más fácilmente la dinámica valvular, aún así es excepcional una manifestación con obstrucción intermitente al flujo, siendo un dato de gravedad e intervención inmediata, ya que puede ser premonitorio de obstrucción aguda grave (8). El estudio doppler mitral mantenido permite apreciar un retardo variable en la apertura del obturador, manifestado como ausencia o disminución significativa del flujo, seguido de la apertura completa con aumento de este, siendo un dato clave para el diagnóstico (8).

El tratamiento de elección en casos seleccionados, con bajo riesgo embólico o alto riesgo quirúrgico, es la fibrinolisis, con unas tasas de éxito del 83\%, mortalidad del $6 \%$ y embolismo sistémico del 12\% $(9,10)$. En los casos con bajo riesgo quirúrgico, contraindicación para fibrinolisis o sospecha de disfunción intrínseca es el reemplazo valvular la opción terapéutica más adecuada, dependiendo la mortalidad de la clase funcional en el momento de la intervención (11).

La presentación tan excepcional del caso que nos ocupa, con una exploración física tan florida, nos hace insistir en la importancia de la valoración inicial del presíncope/síncope ya que nos permitirá llegar a un diagnóstico clínico certero con escasos recursos diagnósticos en la mayoría de las ocasiones. 


\section{Bibliografía}

1. Brignole M. (Chairman), et al.Guidelines on management (diagnosis and treatment) of syncope .Task Force on Syncope, European Society of Cardiology: Eur Heart J 2001; 22: 1256-306.

2. Kapoor W, Syncope N. N Engl J Med 2000; 343: 1856-62.

3. García J, Llamas C, García A, et al. Pronóstico del presíncope en pacientes con cardiopatía estructural. Rev Esp Cardiol 2004; 57: 629-34.

4. Aspas D, Samperiz R, Calvo P, et al. Síncope ¿muchos estudios y pocos diagnósticos? Rev Clin Esp 2004; 204: 9-13.

5. Vongpatanasin W, Hillis LD, Lange RA. Prosthetic heart valves. N Engl J Med 1996; 335: 407-416.

6. Cannegieter SC, Rosendaal FR, Briet E. Thromboembolic and bleeding complications in patients with mechanical heart valve prostheses. Circulation 1994; 89: 635-41
7. Valoración racional de la disfunción de prótesis valvulares. Angel Romero. Archiv Cardiol Mex 2003; 74 (Supl. 2): 60.

8. Romero A, Victoria JL, Franco G, et al. Diagnóstico ecocardiográfico de la disfunción intermitente de prótesis de disco mitral. Arch Cardiol Mex 2001; 71: S3-80.

9. Astengo D, Badano L, Bertoli D. Recombinant tissue activator for Prosthetic mitral valve thrombosis. N Engl J Med 1995; 333: 259

10. Lengyel, et al. Guidelines for management of left sided prosthetic valve thrombosis: a role for thrombolytic therapy. J Am Coll Cardiol 1997; 30: $1521-6$

11. Azpitrate J, Sánchez J, Urda T, et al. Trombosis valvular protésica: ¿Cuál es la terapia inicial más apropiada? Rev Esp Cardiol 2001; 54: 1367-76. 